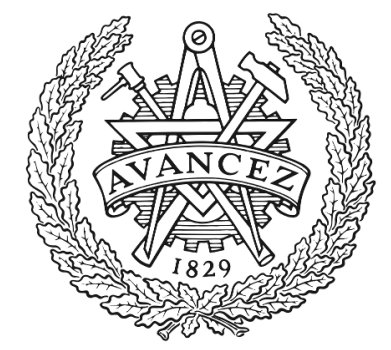

CHALMERS

UNIVERSITY OF TECHNOLOGY

\title{
Low-Profile Ultra-Wideband Directional Dipole Antenna as a Feed for Reflectors in Radio Telescopes
}

Downloaded from: https://research.chalmers.se, 2023-04-26 09:50 UTC

Citation for the original published paper (version of record):

Xie, C., Yin, J., Li, X. et al (2016). Low-Profile Ultra-Wideband Directional Dipole Antenna as a Feed for Reflectors in Radio

Telescopes. 9th International Conference on Microwave and Millimeter Wave Technology

Proceedings, Vol. 1, (ICMMT 2016): 495-497. http://dx.doi.org/10.1109/ICMMT.2016.7761819

N.B. When citing this work, cite the original published paper. 


\title{
Low-Profile Ultra-Wideband Directional Dipole Antenna as a Feed for Reflectors in Radio Telescopes
}

\author{
Chao Xie, Jungang Yin*, Xiang Li \\ Department of Electronics, Hunan University, Changsha, \\ 410082, China, yinjungang126@126.com \\ Feng Pang \\ National Astronomical Observatories of Chinese Academy of Sciences, \\ University of Chinese Academy of Sciences, Beijing, China \\ Jian Yang \\ Antenna Group, Chalmers University of Technology, Gothenburg, S412-96, Sweden
}

\begin{abstract}
In this paper, a small top plate is found useful to improve the impedance bandwidth of an ultra-wideband dipole antenna horizontally above a ground plane. A linearly-polarized prototype based on this new and simple design methodology can operate over nearly 3.5:1 bandwidth with return losses better than $10 \mathrm{~dB}$, and with nearly stable radiation patterns, high $\mathrm{BOR}_{1}$ efficiency and aperture efficiency over the entire operating band.
\end{abstract}

Index Terms - Ultra-Wideband, Dipole Antenna, Top Plate, Radio Telescopes.

\section{INTRODUCTION}

The ground plane of an antenna is typically made by flat conducting plate. In order to get stable directional radiation characteristics and high peek gains, the spacing between the radiator and the ground is about a quarter-wavelength. However, it is tricky to determine the spacing if the ultra-wideband performance is required. Therefore, the radiator is often tilted with an optimum angle from the ground plane, leading to an increase on the antenna height, as can be seen in the Eleven feed [1]-[2] and the self-grounded bow-tie antenna [3]. An alternative way to make a low-profile antenna is to incorporate a special texture on the ground surface, which is possible to alter its electromagnetic properties [4]. By introducing such a high impedance structure, the ground plane does not support surface waves and the interference between the ground and the radiating antenna is eliminated, so the directional radiation patterns can thus be achieved. Based on this idea, a reflector with high-impedance surface was designed in [5]. However the impedance bandwidth is very limited. In [6]-[7] the stable directional radiation patters cannot be obtained over the whole operating band and the impedance bandwidth is only $2: 1$. The paper [8] presented a novel ultra-wideband dipole with a corrugated-reflector, the antenna can operate from 2.75 to $8.35 \mathrm{GHz}$ (3:1 bandwidth) with $\operatorname{VSWRs}<2.0$, but the radiation patterns become poor upwards from $5.0 \mathrm{GHz}$.

To solve this problem, a novel and simple way to design ultra-wideband antenna with stable directional radiation patterns is proposed in this paper. Adding a small metal plate above an ultra-wideband radiator with an ordinary ground plane, it is possible to get stable directional radiation patterns over about 3.5:1 bandwidth. The simulated results shows that the paper provides a new design methodology for the future low-profile ultra-wideband directional antenna without using complex periodical structures on the ground plane.

The Tianlai Project in China [9] is developing a dedicated radio telescope for the dark energy detection [10]-[11]. The feed of the telescope must be able to operate from 400 to 1400 $\mathrm{MHz}(3.5: 1$ bandwidth) with low VSWRs $(<2.5)$ and should have an isolation of at least $20 \mathrm{~dB}$ between two polarization ports. At the same time, low cross polarization, good radiation performance and stable phase center are also required. Based on the proposed linearly-polarized prototype, it is very promising to develop a dually-polarized UWB feed for the Tianlai project.

\section{THE ANTENNA Design}

The proposed antenna is modeled and optimized by CST MWS. The geometry of the source dipole [12] and the entire antenna is shown in Fig. 1 and Fig. 2, respectively; all the dimensions are listed in Table I.

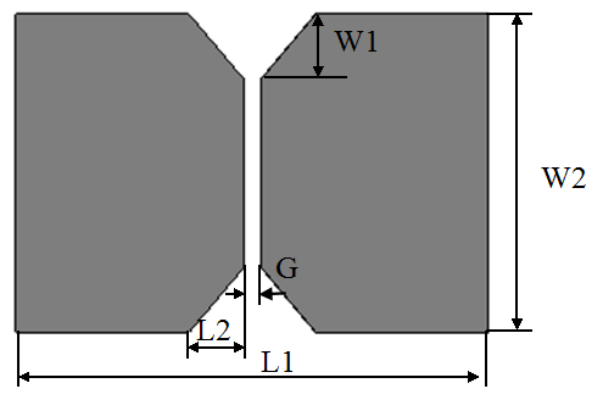

Fig.1. Geometry of the source dipole. 


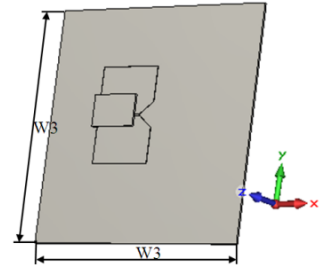

(a)

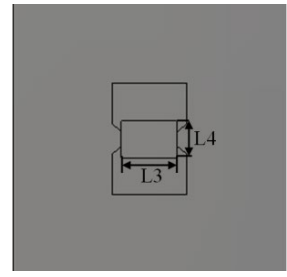

(b)

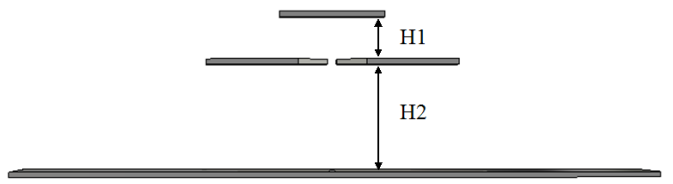

(c)

Fig.2. Geometry of the proposed antenna prototype: (a) 3D view, (b) Front view (c) Side view.

TABLE I . THE PARAMETERS OF THE ANTENNA PROTOTYPE

\begin{tabular}{|c|c|c|c|}
\hline Parameter & Value (mm) & Parameter & Value (mm) \\
\hline H1 & 9 & L3 & 24 \\
\hline H2 & 20 & L4 & 16 \\
\hline L1 & 48.8 & G & 0.8 \\
\hline L2 & 6 & W1 & 7.5 \\
\hline W2 & 32 & W3 & 120 \\
\hline
\end{tabular}

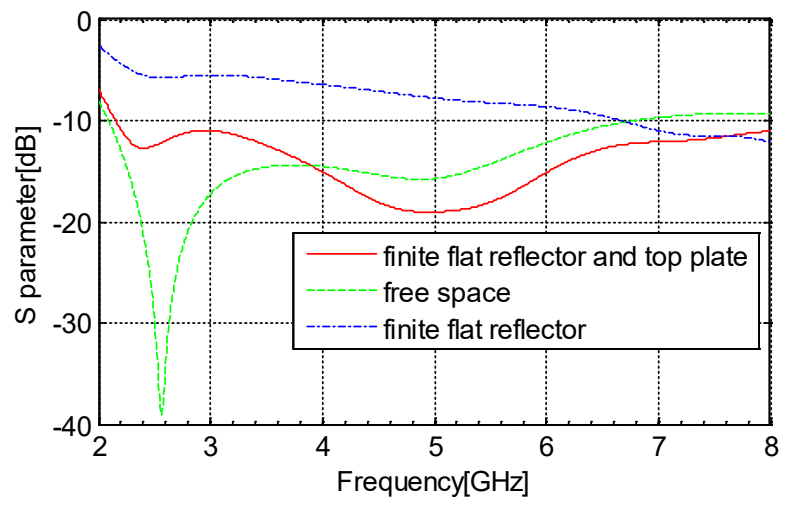

Fig.3. Simulated S-parameters.

\section{Simulation Results}

The simulated S-parameters ( $50 \Omega$ reference) of the evolved models are plotted in Fig. 3. First, the source dipole alone can operate over $2.0-6.5 \mathrm{GHz}$ in free space without a reflector. Second, when the source dipole is placed above a finite ground plane $\left(1.6 \lambda_{4 \mathrm{GHz}} \times 1.6 \lambda_{4 \mathrm{GHz}}\right)$ with a spacing of 12.5 $\mathrm{mm}\left(1 / 4 \times \lambda_{6 \mathrm{GHz}}\right)$, the return loss becomes much worse, which implies that the reflector makes significant influence on the impedance matching. Finally, good matching can be obtained again over $2.0-6.5 \mathrm{GHz}$ by using a top plate, where the lengths of $\mathrm{H} 1$ and $\mathrm{H} 2$ can be adjusted for optimization.
Simulated radiation patterns of the antenna prototype are illustrated in Fig.4. As can be observed, relatively stable directional radiation patterns are obtained over the whole band and the cross-polarization level is below $-25 \mathrm{~dB}$ at the same time. The result in Fig. 6 reveals that the antenna achieves a stable high gain from 8 to $10 \mathrm{dBi}$.

Fig. 5 shows the aperture efficiency and its subefficiencies

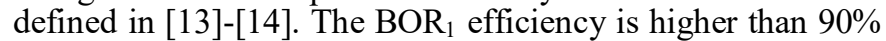
and the aperture efficiency is higher than $50 \%$ over the whole band 2.0-6.5 GHz.

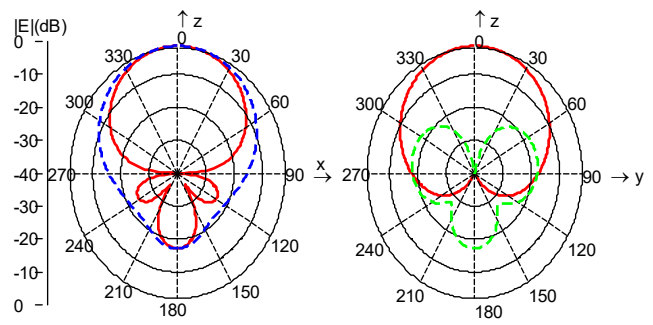

(a) $2.1 \mathrm{GHz}$

(b)

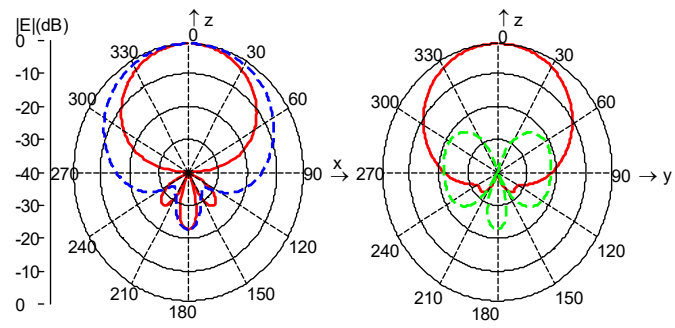

(a) $3.5 \mathrm{GHz}$

(b)

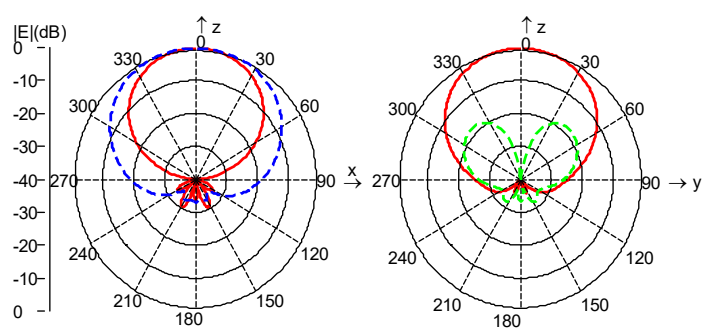

(a) $5.0 \mathrm{GHz}$

(b) 


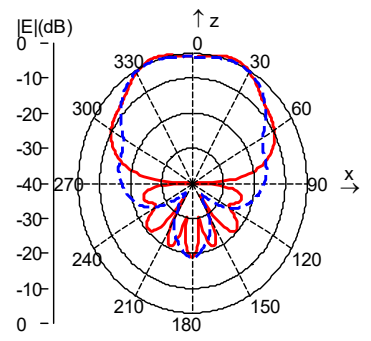

(a)

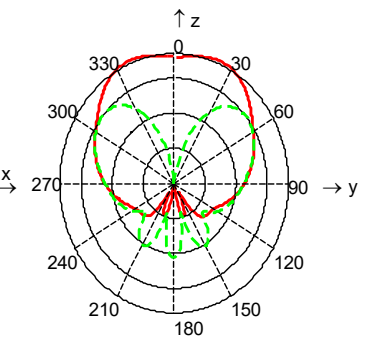

(b)
Fig .4. Simulated radiation patterns at different frequencies: (a) - simulated E-plane (zoy-plane), ---- simulated H-plane (zox-plane). (b) — simulated co-pol. and -----simulated cross-pol. at $+45^{\circ}$ plane.

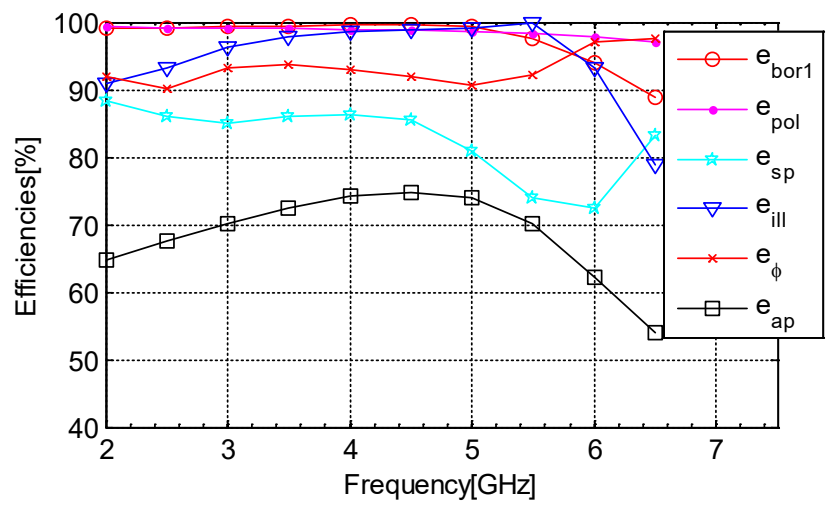

Fig.5. Simulated aperture efficiency and its subefficiencies.

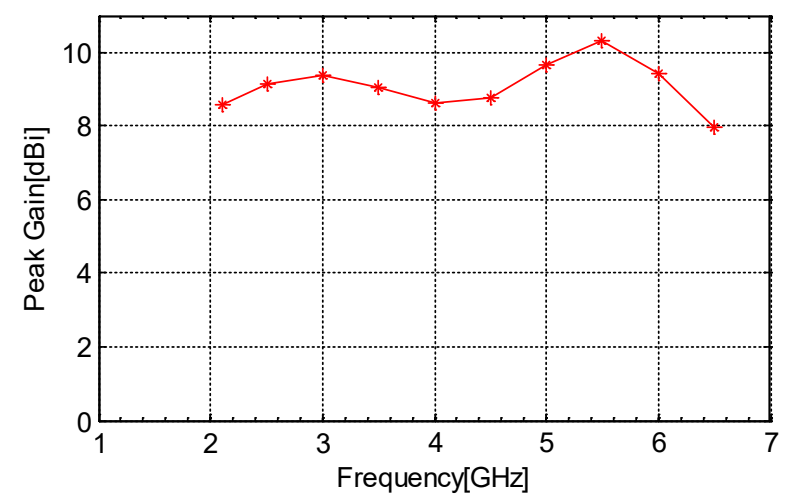

Fig.6. Simulated gain of the antenna.

\section{CONCLUSION}

A simple low-cost low-profile ultra-wideband directional dipole antenna is proposed in this paper. Merely with a well-designed top plate, a horizontal flat dipole radiator can operate over an ultra-wide bandwidth above an ordinary solid ground plane. Based on this idea, a linearly-polarized model is studied by simulations and calculations. The linearlypolarized prototype can operate over $2.0-6.5 \mathrm{GHz}$ (nearly 3.5:1 bandwidth) with return losses better than $10 \mathrm{~dB}$, and with aperture efficiencies better than $50 \%$. The fabrication and measurement of the antenna will be carried out in the near future.

\section{REFERENCE}

[1] J. Yang, M. Pantaleev, P.-S. Kildal, B. Klein, Y. Karandikar, L. Helldner, N. Wadefalk, and C. Beaudoin, "Cryogenic 2-13GHz eleven feed for reflector antennas in future wideband radio telescopes," IEEE Trans. Antennas Propag., vol. 59, no. 6, pp. 1918-1934, Jun. 2011.

[2] J. Yin, J. Yang, M. Pantaleev and L. Helldner, "The circular eleven antenna: a new decade-bandwidth feed for reflector antennas with high aperture efficiency," IEEE Trans. Antennas Propag., vol. 61, no. 8, pp. 3976-3984, Aug. 2013.

[3] H. Raza, A. Hussain, J. Yang and P.-S. Kildal, "Wideband compact 4-port dual polarized self-grounded bowtie antenna," IEEE Trans. Antennas Propag., vol. 62, no. 9, pp. 4468-4473, Sep. 2014.

[4] H. Raether, "Surface plasmons on smooth and rough surfaces and on gratings," Berlin, Germany, Springer-Verlag, 1998.

[5] D. Sievenpiper, Lijun. Zhang, R. F. J. Broas, N. G. Alexopolous, E. Yablonovitch, "High-Impedance electromagnetic surfaces with a forbidden frequency band," IEEE Trans. Microw. Theory Tech., vol. 47 , no. 11, pp. 2059- 2074, Nov. 1999.

[6] L. Akhoondzadeh-ASL, D.J. Kern, P.S. Hall and D.H. Werner, "Wideband dipoles on electromagnetic bandgap ground planes," IEEE Trans. Antennas Propag., vol. 55, no. 9, pp. 2426 -2434, Sep. 2007.

[7] V. V. Yem and T. T. Phuong, "Ultra-wideband low-profile spiral antennas using an EBG ground plane," The 2010 international conference an advanced technologies for communications, pp. 89-94, 2010.

[8] Qi Wu, Ronghong. Jin, Junping. Geng and Donglin. Su, "On the performance of Printed dipole antenna with novel composite corrugated-reflectors for low-profile ultra-wideband applications," IEEE Trans. Antennas Propag., vol. 58, no. 12, pp. 3839-3846, Dec. 2010.

[9] X. L. Chen, International Journal of Modern Physics: Conference Series, 2012, 12(01):256.

[10] Riess. A, et al. Astronomical Journal, 116:1009, 1998.

[11] Perlmutter. S, et al. Astrophysical Journal, 517:565, 1999.

[12] J. M. Qiu, Z. W. Du, J. H. Lu and K. Gong, "A case study of improve the impedance bandwidth of a planar monopole," Microw. Opt. Technol. Lett., vol. 45, no. 2, pp. 124-126, Apr. 2005.

[13] P.-S. Kildal, "Factorization of the feed efficiency of paraboloids and cassegrain antennas," IEEE Trans. Antennas Propag., vol. 33, no. 8, pp. 903-908, Aug. 1985.

[14] P.-S. Kildal and Z. Sipus, "Classification of rotationally symmetric antennas as types BOR0 and BOR1," IEEE Trans. Antennas Propag., vol. 37, no. 6, pp. 114-117, Dec. 1995. 